or two doses of an antibiotic, however, withholding treatment that will reduce a three to five day illness to one which lasts only 12 to 24 hours is difficult. Travellers and their medical advisors should therefore develop self treatment strategies that should include advice about early oral rehydration therapy together with the self administration of loperamide and antibiotics. Medical advice, however, should be sought for severe diarrhoea and dehydration, dysentery, and persistent diarrhoea.

Prevention is always better than treatment, but sadly the standard advice for preventing travellers' diarrhoea almost always fails. Formal evaluation of Swiss travellers visiting Africa and Asia indicated that only $2 \%$ were able to consistently adhere to the strict dietary advice given. ${ }^{9}$ Even when travellers are careful they can often be caught out. Bacterial enteropathogens are able to survive in food that is too hot to touch $\left(50^{\circ} \mathrm{C}\right)^{10}$ and bacteria in ice cubes can be recovered from cocktails containing tequila, whisky, and other spirits. ${ }^{11}$

For many business travellers it is often impossible and indeed unsociable not to eat with local residents and strict control of food and beverage intake may therefore be difficult. Various antibiotics taken prophylactically will prevent travellers' diarrhoea, but most experts would not recommend this for all travellers. People at high risk of acquiring travellers' diarrhoea or with increased susceptibility to its effects might consider prophylaxis, as could the short term visitor for whom the loss of even 12 to 24 hours would seriously interfere with their visit.

Economic arguments have been produced for more liberal use of antibiotic prophylaxis, although their effects would probably militate against this. Alternative preventive measures such as bismuth preparations might also be considered, although their efficacy compares unfavourably with that of antibiotics. Work continues on vaccines to prevent travellers' diarrhoea, but as yet these are not widely available and require further evaluation before they can be recommended.

M J G FARTHING Professor of gastroenterology

Department of Gastroenterology,

St Bartholomew's Hospital,

London EC1A 7BE

1 Farthing MJG, Du Pont HL, Guandalini S, Keusch GT, Steffen R. Treatment and prevention of travellers' diarrhoea. Gastroenterology International 1992;5:162-75.

Shlim DR, Cohen MT, Taton M, Rajah R, Long EG, Ungar BL. An algae-like organism associated with an outbreak of prolonged diarrhoea among foreigners in Nepal. An f Trop Med Hyg 1991;45:383-9.

3 Bendall RP, Lucas S, Moody A, Tovey G, Chiodini PL. Diarrhoea associated with cyanobacteriumlike bodes: a new coccidian enteritis of man. Lancet 1993;341:590-2.

Walker A. Swimming - the hazards of taking a dip. $B M 7$ 1992;304:242-5.

5 Balarajan R, Raleigh VS, Yuen P, Wheeler D, Machin D, Cartwright R. Health risks associated with bathing in seawater. $B M 7$ 1991;303:1444-5.

Marine Conservation Society. Heinz good beach guide. A guide to over 500 of Britain's cleanest beaches. London: Vermilion, 1993.

7 Farthing MJG. Prevention and treatment of travellers' diarrhoea. Alimentary Pharmacology and Therapeutics 1990;5:15-30.

8 Du Pont HL, Ericsson CD, Mathewson J, Marani S, Knellwolf-Cousin A-L, Martinz-Sandoval FG. Zaldaride maleate, an intestinal calmodulin inhibitor, in the therapy of travellers' diarrhoea.

Kozicki M, Steffen R, Schar M. "Boil it, cook it, peel it or forget it": does this rule prevent travellers' diarrhoea? Int $\mathcal{F}$ Epidemiol 1985; 14:169-72.

10 Bandres JC, Mathewson JJ, Du Pont HL. Heat susceptibility of bacterial enteropathogens. Arch Intern Med 1988;148:2261-3.

1 Dickens DL, Du Pont HL, Johnson PC. Survival of bacterial enteropathogens in the ice of popular drinks. fAMA 1985;253:3141-3.

\title{
Jaundice persisting beyond 14 days after birth
}

\section{Requires immediate referral to a paediatrician}

This week the Children's Liver Disease Foundation launches a campaign to improve the early diagnosis of babies with liver disease. Extrahepatic biliary atresia is the commonest life threatening hepatobiliary disorder in childhood, affecting between one in 14000 and one in 21000 live born infants. 'It must be considered in any infant remaining jaundiced beyond 14 days of age $\mathrm{e}^{2}$ as the success of treatment depends on early surgery. In this condition the urine is always yellow and the stools acholic. Occasionally in infants with atresia the stools are pigmented with yellow or green pigment in the first weeks after birth before bile flow is completely obstructed. Biliary atresia is not a congenital malformation per se but an acquired progressive disease of the biliary system of unknown aetiology.

Despite many reports emphasising the need for early surgical intervention delayed referral remains the pattern for most cases. Long term survival of patients who have undergone surgical treatment early in infancy has been one of the most important advances in paediatric surgery in the past three decades.

From Thomson's first description of the condition in $1891^{3}$ until Kasai's portoenterostomy ${ }^{4}$ most cases were considered uncorrectable. Kasai's procedure, in which a Roux-en-Y loop of jejunum is anastomosed to transected, microscopic bile duct remnants in the porta hepatis, was described in 1959 but not accepted as a standard procedure in Britain until the '70s. It remains the initial choice for surgical management, providing effective bile drainage when performed in the first 2 to 3 months of life.

The development of liver transplantation has further increased the chances of curing biliary atresia. There has been debate over whether orthotopic liver transplantation or the Kasai procedure should be the first treatment tried, but most paediatric surgeons accept that Kasai's portoenterostomy is the preferred treatment. Transplantation is not jeopardised by a previous portoenterostomy and should probably be reserved for those in whom portoenterostomy fails initially or who deteriorate with progressive liver disease some months or years later. The five year survival rate of portoenterostomy is $50 \%$ to $60 \%$. The survival rate of liver transplantation in children less than 1 year of age is similar, ${ }^{5}$ although the overall survival rate in all children is over $70 \%$. It must be remembered, however, that orthotopic liver transplantation is not without attendant risks of life long immunosuppression and complications. ${ }^{6}$

All infants presenting with biliary atresia below 12 weeks of age should undergo a Kasai portoenterostomy, but beyond 12 weeks some centres immediately consider liver transplantation. The need for transplantation can also be determined by measuring the size of ductuli at the porta hepatis. ${ }^{7}$ The larger the ductuli at the porta hepatis the better the prognosis. Early recognition of the condition and early referral for surgery can reduce the number of infants requiring transplantation. Many series have shown that infants operated on before 2 months of age have a $90 \%$ chance of achieving satisfactory bile drainage. Each week after this reduces the success rate, and few cases have been successfully operated on beyond 3 months of age. The preoperative investigations to exclude other causes of jaundice take about two weeks. The following guidelines ${ }^{8}$ should be observed: 
All infants jaundiced after 2 weeks of age must have their urine tested for bilirubin and their total and direct bilirubin measured.

If conjugated bilirubin is present the infant should be referred to a paediatrician for urgent investigation.

The doctor should see the stool to determine whether it is yellow or green; if it lacks pigment the infant should be referred to a specialist centre to exclude or treat biliary atresia.

Introducing systematic screening for hepatobiliary disorders has been suggested, as has reducing the age of well baby reviews from 6 to 4 weeks of age. It would allow the identification of infants with hepatobiliary disorders and other conditions that may benefit from earlier diagnosis.

Physiological jaundice almost always clears by 14 days of age except in a very few breast fed infants. In Japan, parents receive written advice on the serious implications of yellow stained urine, pale stools, and jaundice in early infancy. Posters in infant welfare clinics and general practitioners' surgeries reinforce this message. In Japan well baby review is carried out at 4 weeks; other countries should adopt similar measures.
The "Yellow Alert" National Awareness Campaign launched this week is the first serious attempt to tackle the problem in Britain. As well as targeting parents it is aimed at ensuring that all doctors dealing with babies are fully aware of liver disease, its signs, and the need for early intervention.

GORDON A MACKINLAY

Consultant paediatric surgeon

Royal Hospital for Sick Children,

Edinburgh EH9 1LF

1 McClement JW, Howard ER, Mowat AP. Results of surgical treatment for extrahepatic biliary atresia in United Kingdom 1980-2. BMF 1985;290:345-7.

2 Mieli-Vergani G, Howard ER, Portman B, Mowat AP. Late referral for biliary atresia-missed opportunities for effective surgery. Lancet 1989;i:421-3.

3 Thomson J. On congenital obliteration of the bile ducts. Edinburgh Med $\mathcal{f}$ 189 1;37:523-31.

4 Kasai M, Suzuki S. A new operation for "non correctable" biliary atresia; hepatic portoenterostomy. Shujitsu 1959;13:733-9.

5 Sokal EM, Veyckemans F, de Ville de Goyet J, Moulin D, van Hoorebeek N, Alberti D, et al. Liver transplantation in children less than 1 year of age. $\mathcal{F}$ Pediatr 1990;117:205-10.

6 De Conti RW, Craver RD, Willis GW, Hill CB, Hayes DH, Arensman RM. Extrahepatic biliary atresia: from diagnosis to liver transplantation. Paediatr Surg Int 1992;7:337-40.

7 Ohya T, Miyano T, Kimura K. Indication for portoenterostomy based on 102 patients with Suruga II modication. I Pediatr Surg 1990;25:801-4.

8 Hussein M, Howard ER, Mieli-vergani G, Mowat AP. Jaundice at 14 days of age: exclude biliary atresia. Arch Dis Child 1991;66:1177-9.

\section{Clozapine: progress in treating refractory schizophrenia}

\section{Side effects, but a cost-benefit analysis supports treatment}

The rediscovery of the unique properties of clozapine mark an advance in the treatment of acute and chronic schizophrenia. Unlike typical neuroleptics, the relief of symptoms with clozapine is not tightly coupled with unpleasant extrapyramidal side effects and $30 \%$ to $50 \%$ of patients with symptoms unresponsive to typical neuroleptics improve on clozapine.

Clozapine was synthesised in 1958, and a clinical trial in 1962 found that it was highly effective in chronic schizophrenia.' Results from further clinical trials led to its licensing for treatment in over 30 countries. In 1975, however, granulocytopenia occurred in 16 patients in Finland; agranulocytosis developed in 13 of these patients, of whom eight died. Many countries then withdrew clozapine from use.

Its reintroduction, particularly in the United Kingdom and the United States, followed a large trial by Kane and colleagues, which showed that clozapine was more effective than chlorpromazine - both in patients with chronic delusions and hallucinations not fully responsive to standard treatment and in patients with thought disorder and negative symptoms of schizophrenia such as emotional withdrawal, psychomotor retardation, and disorders of affect. ${ }^{2}$ The patients had been selected for this trial if they were refractory to high doses of haloperidol. Over six weeks $30 \%$ of the patients treated with clozapine improved compared with only $4 \%$ of the patients treated with chlorpromazine. Longer trials suggested that up to a half of patients improve after six months' treatment with clozapine. ${ }^{3}$ Some patients whose refractory schizophrenia had kept them in hospital for many months responded to clozapine. ${ }^{34}$ Social functioning also improved in patients who responded to the drug. ${ }^{5}$

Clozapine's actions probably differ from those of typical antipsychotic drugs because of its different effects on central neurotransmitters. The much lower incidence of acute extrapyramidal side effects with clozapine is likely to result from its relatively weak antagonism of striatal dopamine $\mathrm{D}_{2}$ receptors. Importantly, the side effect of tardive dyskinesia does not seem to occur, even with long term use. This may be explained by the failure of chronic long term clozapine treatment to suppress the release of striatal dopamine, but clozapine's relatively strong blocking effect on serotonin $\mathrm{S}_{2}$ receptors may be equally important. ${ }^{6}$ Patients with low ratios of homovanillic acid to 5-hydroxyindoleacetic acid in their cerebrospinal fluid respond better to clozapine ${ }^{7}$ which is consistent with the hypothesis that what is important for the therapeutic actions of the drug is the balance between dopaminergic and serotoninergic neurotransmitter systems. In particular, compared with typical antipsychotic drugs, clozapine causes a greater antagonism of serotonin $S_{2}$ receptors relative to $D_{2}$ receptors ${ }^{6}$; the standard neuroleptic drugs are thought to act through blocking $\mathrm{D}_{2}$ receptors. Clozapine also has a high affinity for $\mathrm{D}_{4}$ receptors and a relatively high affinity for $\mathrm{D}_{1} .{ }^{689}$ The selective interaction with these different receptors is thought to account for its profile of clinical actions.

Schizophrenic patients most likely to benefit from clozapine are those who have not responded to other antipsychotic drugs and those beginning to show serious parkinsonian side effects or signs of tardive dyskinesia. Some patients respond well to low doses-for example, $50 \mathrm{mg}$ a day.

Sandoz Pharmaceuticals was granted a product licence for clozapine (Clozaril) in Britain in 1989 for use in treatment resistant schizophrenia. Owing to the relatively high risk of blood dyscrasia, the licensing requirements included the stipulation that clozapine must be started only in inpatients and that its use should be restricted to patients registered with the Clozaril Patient Monitoring Service, which provides regular haematological screening to a defined standard. Patients' blood samples need to be sent to the monitoring service at the following times: before the start of treatment with clozapine, weekly during the first 18 weeks of treatment, and fortnightly thereafter. The monitoring service detects early falls in the neutrophil count, and prescriptions for clozapine cannot be issued until the monitoring service has cleared the haematological results. If neutropenia occurs prompt discontinuation of clozapine allows the neutrophil 